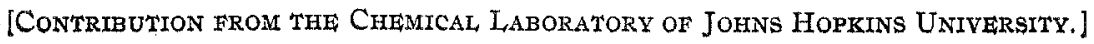

\title{
HEAT OF WETTING OF SILICA GEL.
}

\author{
By W. A. Patrick and F. V. Grimm.
}

Received June 25,1921 .

The present investigation was undertaken with a view to ascertaining whether or not it was possible to account for the heat of wetting of silica gel on the basis of the changes of surface energy involved. There is available considerable experimental material, $1,2,3$ relative to the heats of adsorption and wetting with adsorbents other than silica gel, and the interpretation of the results has been usually that of an effect due to the compression of the adsorbed liquids. The strongest evidence in favor of this view has been the fact that the heat of wetting by water was found to be negative between $0^{\circ}$ and $4^{\circ}$.

Inasmuch as our ideas of the mechanism of adsorption have emphasized the attractive forces between the molecules of the adsorbed substance rather than that between the latter and the adsorbent, it was very difficult for us to accept the above explanation of the heat of wetting. We accordingly planned to measure the heats of wetting of silica gel by a number of liquids with a view to ascertaining whether the observed results were in accord with the involved changes in surface energy.

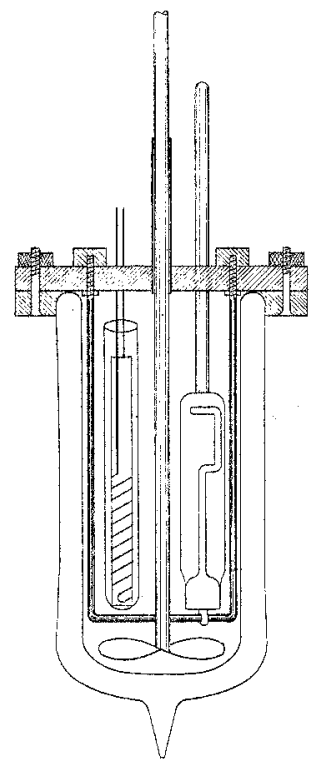

Fig. 1.

It was our belief that by using an adsorbent such as silica gel we could observe an easily measured heat of wetting by a material of definite and known composition. Furthermore inasmuch as the surface presented by such a gel is a water surface, we would be in a position to evaluate the changes in surface energy involved provided the interfacial surface tensions and their temperature coefficients were known.

The calorimeter used in these measurements was a $150 \mathrm{cc}$. Dewar bulb fitted tightly with a hard rubber cover. Although the main portion of the heat of wetting developed within 5 minutes, nearly an hour was required for the calorimeter to give constant readings. For this reason it was thought best to carry out the measurements adiabatically. The calorimeter was accordingly placed in a wooden box which was provided with a fan for stirring the air, and

\footnotetext{
1 Jungk, Pogg. Ann., 125, 292 (1865).

${ }^{2}$ Schwalbe, Ann. Physik., 16, 32 (1905) (Bibliography).

${ }^{3}$ Lamb and Coolidge, This Journal, 42, 1146 (1920).
} 
electric lamps for heating purposes. It was found that by working with a constant room-temperature of about one degree below that at which the measurement was made it was possible to keep the temperature of the air-bath to within $0.01^{\circ}$ or $0.02^{\circ}$ of the temperature of the calorimeter. By this arrangement the lass due to radiation was minimized.

The silica gel was placed inside the calorimeter before mixing with the liquid, thus insuring an initial constant temperature. The arrangement by means of which this was accomplished is best seen from the drawing (Fig. 1).

The vessel which contained the gel was provided with a carefully ground glassstopper. Upon turning and lifting the glass rod sealed to the bottom of the vessel, the gel was thrown into the liquid. A glass hook attached to the stopper enabled the gel to be completely removed from the bottle.

A light stirrer made of glass and turned at a speed of about $500 \mathrm{r} . \mathrm{p}$. m. gave very satisfactory results. A correction of from $0^{\circ}$ to $0.003^{\circ}$ per minute was applied to take care of the heat due to the stirring alone. The correction varied with the different liquids and was determined separately for each measurement.

The heating unit used for determining the thermal capacity of the calorimeter was constructed similarly to the one used by 'Tucker ${ }^{4}$ in his heat of dilution experiments. It consisted of a Nichrome wire wound around a thin strip of mica and immersed in a thin-walled glass tube filled with oil. Copper leads were brazed on the resistance wire and the former alone extended out of the calorimeter.

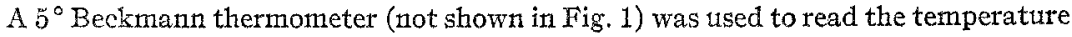
of the calorimeter and could be read to within $0.002^{\circ}$. A similar thermometer was placed in the air-bath.

The thermal capacity of the calorimeter was determined with the same charge in the calorimeter after a measurement of the heat of wetting by water had been made. Proceeding in this manner it was unnecessary to know the specific heat of the gel. An electric current from a storage battery was passed through the heating unit for a period of 5 minutes, the exact time being determined with a stop-watch. The resistance of the coil was determined and found to be 1.475 ohms. Current was measured with a milliammeter, readings being taken every 15 seconds. The thermal capacities of liquids other than water were calculated from their specific heats and densities. In all the experiments the same volume of liquid was used, namely, $50.07 \mathrm{cc}$. Furthermore, conditions were so arranged as to bring the final temperature to $25^{\circ}$ in each measurement.

TABLE I.

\begin{tabular}{|c|c|c|c|c|}
\hline $\begin{array}{l}\text { Resistance } \\
\text { of coil. }\end{array}$ & $\begin{array}{l}\text { Time. } \\
\text { Seconds. }\end{array}$ & $\begin{array}{l}\text { Amperes. } \\
\text { Average. }\end{array}$ & $\begin{array}{c}\text { Temperature } \\
\text { increase. }\end{array}$ & Cal./degree \\
\hline 1.475 & 270.6 & 1.0717 & 1.728 & 63.46 \\
\hline 1.475 & 300.0 & 1.0920 & 1.987 & 63.53 \\
\hline 1.475 & 300.0 & 1.0701 & 1.910 & 63.44 \\
\hline
\end{tabular}

Av. $\quad 63.48$

\footnotetext{
${ }^{4}$ W. S. Tucker, Trans. Roy. Soc, 215, 319 (1915).
} 
Taking 0.99681 as the density and 0.99765 as the specific heat of water at $25^{\circ}$ the water equivalent of the calorimeter becomes $13.686 \mathrm{cal} . /$ degree.

The following values of specific heat and density at $25^{\circ}$ were used in the calculation of the thermal capacity of the other liquids.

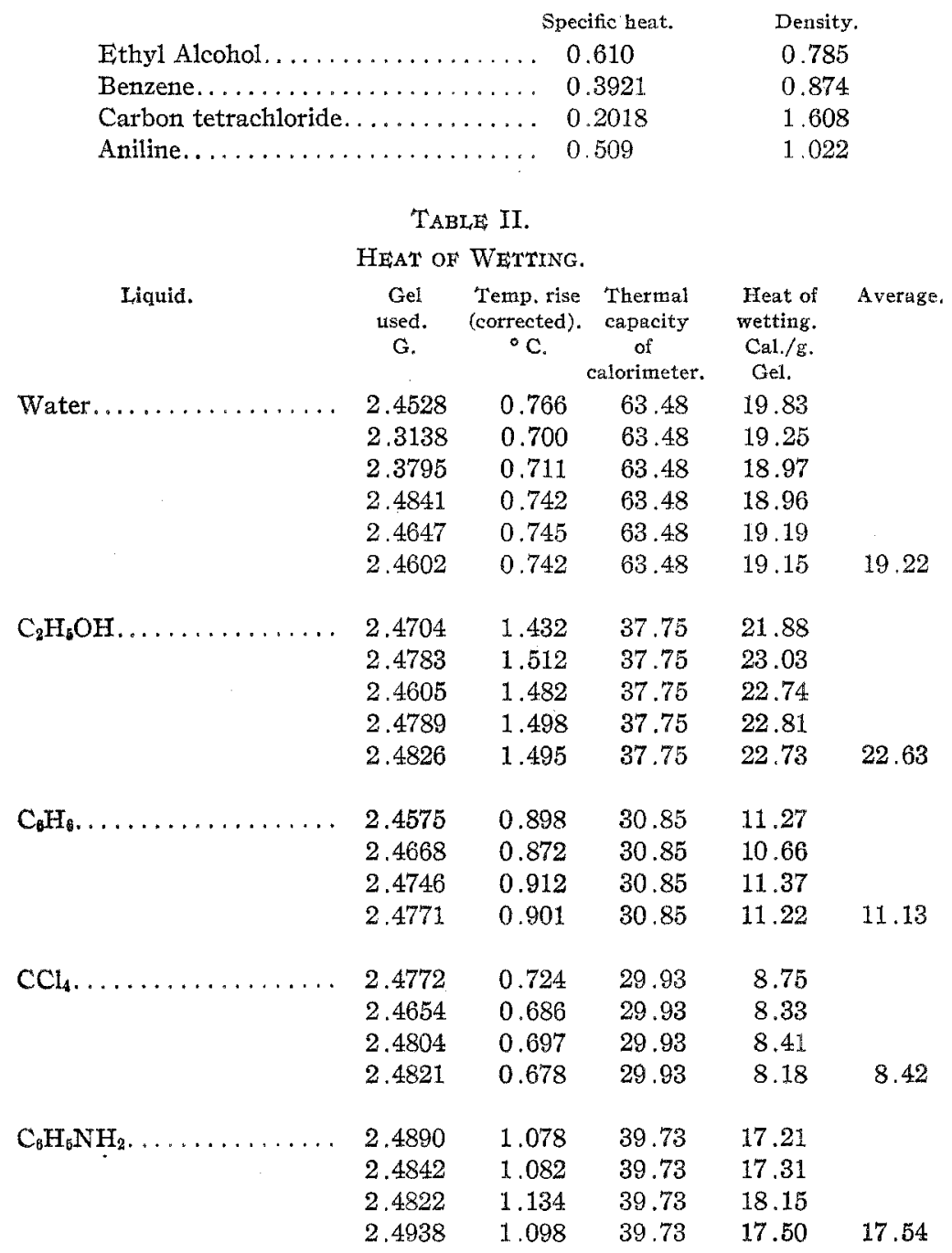

The heat of wetting by water was also determined between $0^{\circ}$ and $4^{\circ}$ and in all cases the results were positive. At $2.86^{\circ}$ with $2.48 \mathrm{~g}$. of gel the temperature increased to $3.92^{\circ}$ within the first 5 minutes. Due to the fact that we did not try to make the calorimeter adiabatic, no effort was made to secure an exact value of the heat of wetting at this temperature. 
With aniline the wetting was much slower and required about 2 hours before the temperature readings were constant.

\section{Discussion.}

The fact that the heat of wetting by water between $0^{\circ}$ and $4^{\circ}$ was found to be positive is in itself considerable evidence against the compression theory of the heat of wetting.

There remains however the fact that a number of investigators have shown that the heat of wetting is negative between $0^{\circ}$ and $4^{\circ}$. On closer examination it is evident that such experimentors were dealing with an effect of an entirely different order of magnitude. For example G. Schwalbe ${ }^{2}$ in order to detect the small heats of wetting, employed a thermocouple and a galvanometer of such sensitivity that $1^{\circ}$ change in temperature corresponded to $1.86 \times 10^{-7} \mathrm{amp}$. In our opinion such investigations were not dealing with capillary adsorption at all, but were measuring the heat of adsorption on a plane surface. The initial film of adsorbed material is undoubtedly under compression, but the amount of liquid in such a state is negligible as compared with the condensation that occurs in a body exinibiting capillary structure.

It occurred to us that it might be possible to explain the above measurements from consideration of surface energy changes. Silica gel in our opinion consists of an agglomeration of silica nuclei, each particle being surrounded with an envelope of water. Therefore the gel may be regarded as exhibiting an enormous water surface which is the seat of considerable energy. When this material is immersed in water the above surface disappears due to the fact that a water-water interface cannot exist. When on the other hand, the gel is wetted by a liquid such as benzene the total surface undergoes no diminution in area, but the surface energy is greatly decreased due to the diminution of the interfacial tension. At the same time an amount of heat is set free equivalent to the decrease in surface energy, thus accounting for the heat of wetting.

Inasmuch as the total surface energy is given by the following expression

$$
E=\omega \sigma-T \frac{\mathrm{d} \sigma}{\mathrm{d} t} \omega
$$

where $\omega$ is the surface and $\sigma$ the surface tension, it is first necessary to ascertain whether the surface of one gram of silica gel is of sufficient magnitude to account for the measured heat effects. The surface tension of water at $25^{\circ}$ is according to Harkins and Cheng 52.05 dynes per cm., and the value of $\mathrm{d} \sigma / \mathrm{d} t=-0.1511$. Therefore $E$ at $25^{\circ}$ equals 117.08 ergs per $\mathrm{cm}^{2}$. In order to obtain a heat of wetting of $19.22 \mathrm{cal}$. per gram of gel it is necessary that the latter exhibit a surface of

W. D. Harkins and X. C. Cheng, This Journal, 43, 35 (1921). 


$$
\omega=\frac{19.22 \times 4.182 \times 10^{7}}{117.08}=6.9 \times 10^{6} \mathrm{~cm} .^{2}
$$

This figure is in good agreement with the ultra-microscopically observed degree of heterogeneity; Zsigmondy observing particles of the order of 5 $\mu \mu$ in diameter. Assuming rotighly the density of the gel particles to be 2 it is evident that the surface exhibited by $1 \mathrm{~g}$. of particles of the above size would be

$$
\frac{3 \times 0.5}{2.5 \times 10^{-7}}=6 \times 10^{6} \mathrm{~cm}^{2}
$$

It is evident from such a calculation that the surface energy is therefore sufficient to explain the observed heat effects.

There still remains to be tested the agreement between the calculated and measured values of the heat of wetting by the other liquids. In the calculation of the heat of wetting, the surface of the gel is assumed to be the same as that evaluated from the heat of wetting by water.

In the case of benzene, for example, the total surface energy after wetting is

$$
E=\omega \sigma^{\prime}-\omega T \frac{\mathrm{d} \sigma^{\prime}}{\mathrm{d} t}
$$

where $\sigma^{\prime}=$ the interiacial tension at the water-benzene surface. The heat of wetting by benzene is equal to the difference between the total surface energy of the gel and the above expression. Using the value given by Harkins for the free surface energy and its variation with the temperature, the total surface energy of $1 \mathrm{~cm}^{2}$ of a water-benzene interface at $25^{\circ}$ is evaluated as $51.66 \mathrm{ergs}$ per $\mathrm{cm} .{ }^{2}$. Inasmuch as a water surface at the same temperature possesses $117.08 \mathrm{ergs}$ per $\mathrm{cm} .{ }^{2}$, it is evident that during the wetting $117.08-51.66$ or 65.42 ergs per $\mathrm{cm}^{2}{ }^{2}$ must have been converted into heat. Furthermore, if in the case of wetting by water the final surface energy was reduced to zero, then the heat of wetting by benzene should be in the ratio of $65.42: 117.08$ to that of the heat of wetting by water, or equal to 10.74 cal. per g. of gel. The heat of wetting observed was, 11.13 cal. per g.

In a similar manner it may be calculated using the data of Harkins that the total interfacial energy of a water-carbon tetrachloride surface at $25^{\circ}$ is equal to $73.86 \mathrm{ergs}$ per $\mathrm{cm}^{2}{ }^{2}$. From this value we calculated that the heat of wetting by carbon tetrachloride should be 7.10 cal. per g; the observed was 8.42 cal. per $\mathrm{g}$.

In the case of aniline no data are available as to the variation of the free interfacial energy with the temperature, and evaluation of the latent surface energy is uncertain. However, we may approximate this value as follows. The interfacial tension of water-aniline at $20^{\circ}$ is given by Harkins as 5.77 dynes per $\mathrm{cm}$. The critical solution temperature of these two liquids is $167^{\circ}$, at which point the interfacial tension must be zero. 
By assuming a straight line temperature function we therefore obtain $\mathrm{d} \sigma^{\prime} / \mathrm{d} t=-0.04$. The bound surface energy at $25^{\circ}$ is then found to be 11.92 ergs per $\mathrm{cm} .{ }^{2}$ which added to the free energy gives a total surface energy of 17.69 ergs per ctn. ${ }^{2}$ for the water-aniline interface. The heat of wetting by aniline may then be calculated to equal 16.56 cal. per $\mathrm{g}$; the observed, was 17.54 cal. per $\mathrm{g}$.

In all cases it is noted that our calculated heats of wetting are lower than the observed values, although the agreement is remarkable considering the nature of the calculations. The discrepancy is, however, easily accounted for, although unfortunately we are not able to reduce our corrections to a quantitative basis. It has been shown by Davidheiser and Patrick ${ }^{b}$ that silica gel contains a certain amount of acid. Furthermore it is known that acids decrease the interfacial tension at the water-benzene boundary. In other words it is decidedly probable that the total surface energy after wetting is lower than that calculated. Furthermore it is possible that the surface energy of the gel is greater than that assumed by us, due to the fact that the adsorbed water layer exhibits a higher surface tension than does ordinary water. Both of these effects would tend to increase the heat of wetting; in our opinion, however, the lowering of the interfacial tension due to the presence of acid is sufficient to account for the entire difference between the measured and calculated values.

In the case of alcohol, at first thought we should expect the heat of wetting to be equal to the heat of wetting by water inasmuch as the water and alcohol are miscible in all proportions. The observed heat of wetting, however, is found to be $3.41 \mathrm{cal}$. per $\mathrm{g}$. higher than that for water. This difference is too great to be explained on the basis of heat of solution of water in alcohol.

The ideas upon which our explanation is based will be developed in more detail in a paper under preparation dealing with the effect of capillarity upon equilibrium. Nevertheless it may be said at this time that it is conceivable that in a small capillary tube water may present a surface against alcohol. Furthermore if such a surface were to possess a positive temperature coefficient of free surface energy, we should then expect the heat of wetting by alcohol to be greater than that of water. For example the heat of wetting of silica gel by heptin may be calculated from the interfacial data of Harkins. According to the latter the total surface energy of a heptin-water surface at $20^{\circ}$ equals $-19.60 \mathrm{ergs}$ per $\mathrm{cm} .{ }^{2}$. We should therefore expect this liquid to exhibit a higher heat of wetting than water, namely 22.4 cal. per $g$. of gel.

\section{Summary.}

1. The heats of wetting of silica gel by water, benzene, carbon tetrachloride, ethyl alcohol and aniline have been determined.

- Davidheiser and Patrick, to be published later. 
2. The heat of wetting of silica gel by water has been found to be positive between $0^{\circ}$ and $4^{\circ}$.

3. The observed heats of wetting have been satisfactorily accounted for on the basis of surface energy changes.

BaLtimore, Maryiand.

[Contribution from the Chemical Laboratory of the University of Michigan.]

\section{THE TRANSFERENCE NUMBERS OF SULFURIC ACID BY THE CONCENTRATION CELL METHOD.}

\section{By Alfred L. Ferguson and Wesley G. France.}

Received June 28, 1921 .

The concentration cell method for the determination of transference numbers was shown, in this laboratory ${ }^{1}$ to be more accurate in the case of uni-univalent electrolytes than the Hittorf method. In the present investigation it is found to apply equally well in the case of the uni-bivalent electrolyte $\mathrm{H}_{2} \mathrm{SO}_{4}$.

The determination involves the measurement of the potentials of a concentration cell without diffusion; a concentration cell with diffusion and reversible with respect to the cation; and a concentration cell with diffusion and reversible with respect to the anion.

The total potential of the concentration cell, reversible with respect to the cation, $\mathrm{Pt}_{\mathrm{H}}\left|\mathrm{H}_{2} \mathrm{SO}_{4} c_{3}\right| \mathrm{H}_{2} \mathrm{SO}_{4} c_{2} \mid \cdot \mathrm{Pt}_{\mathrm{H}}$, consists of the algebraic sum of the two electrode potentials and the potential at the boundary of the solutions. On the assumption that sulfuric acid dissociates into two hydrogen ions and one sulfate ion, the algebraic sum of the electrode potentials is expressed by the well-known formula

$$
E_{1}=\frac{R T}{F} \ln \frac{c_{1}}{c_{2}}
$$

The potential at the liquid boundary is expressed by the formula

$$
E_{B}=\frac{2 U_{c}-U_{a}}{2\left(U_{c}+U_{a}\right)} \frac{R T}{F} \cdot \ln \frac{c_{1}}{c_{z}}
$$

The hydrogen electrode in the concentrated solution is positive with respect to the hydrogen electrode in the dilute solution. At the boundary of the solutions, the sulfuric acid diffuses from the concentrated to the dilute side, and since the hydrogen ion moves faster than the sulfate ion, the dilute side is positively charged with respect to the concentrated. This means that the potential developed at the boundary opposes the potential of the hydrogen electrodes. The total potential of the hydrogen concentration cell is, therefore, expressed by the equation

'A. L. Ferguson, J. Phys. Chem., 20, 326 (1916). 